\title{
Cordonnier Grade 2 Pneumonia or Bronchopneumonia with PaO2 Greater than $65 \mathrm{mmHg}$
}

National Cancer Institute

\section{Source}

National Cancer Institute. Cordonnier Grade 2 Pneumonia or Bronchopneumonia with PaO2 Greater than $65 \mathrm{mmHg}$. NCI Thesaurus. Code C138316.

Any pneumonia or bronchopneumonia with $\mathrm{PaO} 2$ greater than $65 \mathrm{mmHg}$. 